\title{
Diferentes fontes e níveis de selênio sobre o desempenho de frangos de corte
}

\author{
Differents sources and levels of selenium on performance of broilers
}

\author{
Pascoal FUNARI JUNIOR ${ }^{1}$; Ricardo de ALBUQUERQUE ${ }^{1}$; Flávio Rocha ALVES ${ }^{1}$; Vinícius Diogo \\ Azevedo MURAROLLI ${ }^{1}$; Messias Alves da TRINDADE NETO ${ }^{1}$; Evanildo Moreira da SILVA ${ }^{1}$
}

${ }^{1}$ Departamento de Nutrição e Produção Animal. Faculdade de Medicina Veterinária e Zootecnia da Universidade de São Paulo, Pirassununga-SP

\begin{abstract}
Resumo
As pesquisas em nutrição de frangos de corte estão em busca de ajustes que forneçam às aves os nutrientes necessários para um ótimo desempenho produtivo. Neste contexto, a utilização de microminerais orgânicos vem ganhando força e se mostrando uma alternativa para aumentar a produção. O presente estudo teve como objetivo avaliar os efeitos de três fontes e dois níveis de selênio sobre o desempenho de frangos de corte. Foram utilizados 1440 pintos de um dia, machos, criados até os 42 dias. O delineamento experimental foi inteiramente casualizado com seis dietas experimentais (A: 0,15 $\mathrm{mg} / \mathrm{kg}$ Se inorgânico; B: 0,15 mg/kg Se orgânico; C: 0,15 mg/kg Se inorg. + orgânico; D: 0,45 mg/kg Se inorgânico; E: $0,45 \mathrm{mg} / \mathrm{kg}$ Se orgânico; F: 0,45 mg/kg Se inorg.+ orgânico) e seis repetições com 40 aves cada. Foi utilizado um arranjo fatorial $3 \times 2$ e os dados obtidos foram analisados pelo Statistical Analysis System. Quanto ao desempenho, considerando-se o período total de criação, houve efeito do nível de Se sobre o ganho de peso (GP) e ganho médio diário (GMD); a conversão alimentar foi a única variável que apresentou interação entre fonte e nível de Se, sendo que as variáveis de peso somente apresentaram efeito do nível de inclusão, mostrando que fonte e nível de Se são fatores que juntos podem exercer influência sobre o desempenho dos animais.
\end{abstract}

Palavras-chave: Minerais. Micromineral orgânico. Nutrição. Produção de Aves.

\begin{abstract}
The research in nutrition of broiler chickens is searching for adjustments that supply to the birds the necessary nutrients for better productive performance. In this context the use of organic minerals is gaining force and becoming an alternative to increase the production. The objective of the present study was to evaluate the effect of three sources and two levels of selenium on the performance of broiler chickens. This work analysed 1440 young chickens of one day, males, that were created until the 42nd day. The assignment was completely randomized, with six experimental diets (A: $0,15 \mathrm{mg} / \mathrm{kg}$ inorganic; B: $0,15 \mathrm{mg} / \mathrm{kg}$ organic; C: $0,15 \mathrm{mg} / \mathrm{kg}$ inorg. + organic; D: 0,45 mg/kg inorganic; E: $0,45 \mathrm{mg} / \mathrm{kg}$ organic; F: 0,45 mg/kg inorg. + organic) and six repetitions with 40 birds each. It was used a $3 \times 2$ factorial arrangement and the obtained data were analyzed by PROC GLM of SAS. In regard to the performance, considering the total period (42 days), there was an effect of the level of Se on the weight gain (GP) and average daily gain (GMD); the alimentary conversion was the only variable that presented interaction between source and level of Se and the weight variables only presented the effect of the inclusion level, showing that source and level of Se are factors that together can exercise influence on the performance of the animals.
\end{abstract}

Keywords: Minerals. Organic minerals. Nutrition. Production of broilers.

\section{Introdução}

O selênio (Se) é um micronutriente essencial para o crescimento e manutenção das aves. Este mineral possui diversas funções, entre as quais, a regulação da atividade da glutationa peroxidase, dos hormônios da tireoide e prostaglandinas; aumento da eficiência da vitamina E; melhoria na atuação do sistema imune; suporte de funções reprodutivas; e proteção contra metais pesados ${ }^{1}$. A deficiência de
Se pode levar à necrose hepática, redução da quantidade de proteínas, diátese exsudativa, redução na

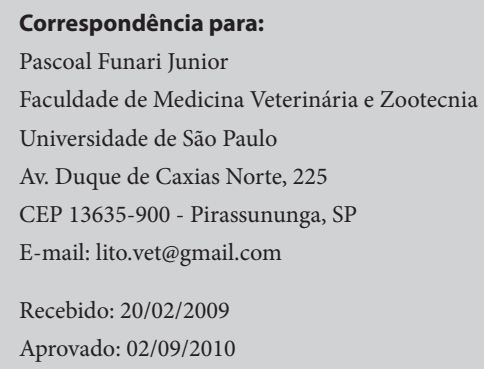


secreção de enzimas digestivas, além de reduzir o crescimento ${ }^{2}$.

A suplementação de Se nas rações de frangos de corte deve ser realizada, pois os solos nacionais em sua maioria são pobres nesse mineral ${ }^{3}$. A forma mais comum de suplementação é o selenito de sódio, forma inorgânica. Já a forma orgânica de suplementação de Se é produzida a partir de uma cultura de levedura (Saccharomyces cerevisae) enriquecida com Se inorgânico. Este processo leva à incorporação de Se ao invés do enxofre na metionina ou cisteína formando selenocisteína ou selenometionina ${ }^{4}$. Enquanto minerais como o sódio, cloro e potássio são completamente absorvidos na forma inorgânica, os microminerais em geral têm taxa de absorção baixíssima ${ }^{3}$. A selenometionina é absorvida do trato digestivo por um mecanismo ativo de transporte similar ao da metionina, enquanto que o selenito não é transportado ativamente $^{5}$ aumentando a taxa de absorção do Se.

O Se pode influenciar a qualidade da carne $\mathrm{e}^{6}, \mathrm{o} \mathrm{em}-$ penamento $^{7}$, a conversão de tiroxina em tri-iodotironina e a imunidade passiva de animais ${ }^{8}$, o efeito tóxico e carcinogênico das aflatoxinas ${ }^{9}$, as funções reprodutivas, o desenvolvimento, a imunocompetência, o envelhecimento ${ }^{10} \mathrm{e}$ a resistência ao calor ${ }^{11}$. Segundo alguns autores ${ }^{6,10,12,13}$ o Se orgânico pode atuar como reserva nos músculos e o inorgânico direto na incorporação da selenocisteína atuando em diversas selenoproteínas.

O Se é essencial na dieta humana e também na dos animais, sendo a suplementação na dieta destes uma alternativa para o fornecimento deste elemento aos humanos. Este fato se deve à deposição do Se em produtos de origem animal (carne, leite, ovos) oriundos de animais alimentados com Se na dieta, melhorando não só a saúde e o desempenho dos animais, mas também levando o Se para a dieta humana ${ }^{14}$. O uso do Se orgânico tem se mostrado mais eficiente do que o Se inorgânico na deposição de Se nos tecidos, sem afetar o desempenho, o rendimento de carcaça ou a atividade da GSH-Px ${ }^{15}$.

O presente trabalho tem o objetivo de investigar os efeitos da variação de níveis e fontes de Se sobre o desempenho de frangos de corte.

\section{Material e Método}

Foram utilizados 1440 pintos de corte de um dia de idade, machos, de linhagem comercial, vacinados no incubatório contra Doença de Gumboro e Doença de Marek e aos 14 dias contra Doença de Newcastle, criados até 42 dias em boxes, no aviário experimental da FMVZ-USP. O manejo e equipamentos utilizados foram semelhantes aos de criação comercial, adaptados às condições experimentais.

O delineamento utilizado foi o inteiramente casualizado, com seis tratamentos, seis repetições/tratamento totalizando 36 unidades experimentais (boxes) com 40 aves/box; e um arranjo fatorial 3x2, com três fontes (orgânica, inorgânica e orgânica + inorgânica) e dois níveis de Se $(0,15 \mathrm{mg} / \mathrm{kg}$ e $0,45 \mathrm{mg} / \mathrm{kg})$. As rações foram preparadas de acordo com os níveis nutricionais praticados na criação comercial de frangos de corte, ajustadas para terem os níveis desejáveis de Se. As rações foram elaboradas à base de milho e farelo de soja, variando em sua composição nutricional segundo a fase de criação: inicial (1 a 21 dias), crescimento (22 a 35 dias) e final (36 a 42 dias), conforme mostra a tabela 1. As dietas experimentais só se diferenciaram quanto ao nível e a fonte de selênio da seguinte maneira: D1: 0,15mg/kg Se inorgânico, D2: 0.15mg/kg Se orgânico, D3: 0.15mg/kg Se 50\% orgânico e 50\% inorgânico, D4: 0.45mg/kg Se inorgânico, D5: $0.45 \mathrm{mg} / \mathrm{kg}$ Se orgânico, D6: $0.45 \mathrm{mg} / \mathrm{kg}$ Se 50\% orgânico e 50\% inorgânico. O Se adicionado às rações foi colocado em substituição ao material inerte (caulin), nas dosagens indicadas em cada tratamento.

$O$ peso das aves e o consumo de ração foram medidos através de pesagens semanais. A morte de ani- 
Tabela 1 - Composição percentual e análise calculada das rações nas diferentes fases de criação

\begin{tabular}{lccc}
\hline \multicolumn{1}{c}{ Ingredientes } & $\begin{array}{c}\text { Inicial } \\
\text { \% }\end{array}$ & $\begin{array}{c}\text { Crescimento } \\
\text { \% }\end{array}$ & $\begin{array}{c}\text { Final } \\
\text { \% }\end{array}$ \\
\hline Milho & 52,26 & 57,11 & 63,7 \\
Farelo de Soja & 40,13 & 34 & 28 \\
Óleo de Soja & 3,52 & 4.9 & 4,5 \\
Sal & 0,35 & 0,35 & 0,35 \\
Calcáreo & 1,24 & 1,6 & 1,6 \\
Fosfato Bicálcico & 1,6 & 1,14 & 0,95 \\
Metionina & 0,24 & 0,21 & 0,18 \\
Suplemento Vit.-Min.(1) & 0,2 & 0,2 & 0,2 \\
Antibiótico & 0,01 & 0,01 & 0 \\
Inerte & 0,45 & 0,48 & 0,52 \\
\hline Total & 100 & 100 & 100 \\
\hline ANÁLISE CALCULADA & & & \\
\hline Energia Metabolizável (Kcal/Kg) & 2950 & 3100 & 3150 \\
Proteína Bruta (\%) & 22,5 & 20,0 & 0,441 \\
Metionina digestível (\%) & 0,553 & 0,496 & 0,698 \\
Metionina + Cistina digestível (\%) & 0,855 & 0,773 & 0,841 \\
Lisina digestível (\%) & 1,127 & 0,981 & 0,611 \\
Treonina digestível (\%) & 0,771 & 0,687 & 0,90 \\
Cálcio (\%) & 0,95 & 0,95 & 0,30 \\
Fósforo Disponível (\%) & 0,45 & 0,35 & \multicolumn{2}{c}{} \\
\hline
\end{tabular}

(1) Vit. A 11280 UI, Vit. D 2724 UI, Vit. E 17,2 mg, Vit. K 1,92 mg, Vit. B 2,01 mg, Vit. B 4,5 mg, Vit. B 2,49 mg, Vit. B $12 \mathrm{mg}$, Ácido Nicotínico $30 \mathrm{mg}$, Pantonato de cálcio $12 \mathrm{mg}$, Biotina 0,099 mg, Ácido Fólico 0,99 mg, Antioxidante $1 \mathrm{mg}$, Iodo 1,0065 mg, Ferro 60 mg, Manganês 70,2 mg, Zinco 70 mg, Cobre 10,05 mg.

mais foi anotada diariamente, pesando-se neste momento a ave morta e o comedouro. A partir destes dados, foram calculadas as seguintes variáveis: peso médio $(\mathrm{P})$, ganho de peso médio $(\mathrm{GP})$, ganho médio diário (GMD), consumo médio de ração (CR), e conversão alimentar (CA) para cada unidade experimental.

Os resultados foram analisados com o auxílio do programa computacional Statistical Analysis System. Os dados, que atenderam às premissas estatísticas, foram submetidos à análise de variância e ao teste de média Tukey a fim de separar o possível efeito de tratamento. Para todas as análises estatísticas foi utilizado nível de significância de 5\%.

\section{Resultados e Discussão}

Os resultados estão apresentados na tabela 2. Foi observado efeito para o fator nível $(\mathrm{p}=0,0225)$, sendo o nível $0,45 \mathrm{mg} \mathrm{Se} / \mathrm{kg}$ o que proporcionou maior peso médio, ganho de peso e ganho médio diário. Este resultado discorda do estudo realizado por Swain, Johri e Majumdar ${ }^{16}$ que utilizaram $0 \mathrm{mg} /$ $\mathrm{kg}, 0,1 \mathrm{mg} / \mathrm{kg}, 0,5 \mathrm{mg} / \mathrm{kg}$ e $1 \mathrm{mg} / \mathrm{kg}$ de Se nas dietas experimentais, e não houve variação no ganho de peso das aves. Dahlke et al. ${ }^{17}$ também não observaram efeito do nível de Se aos 42 dias. Moreira et al. ${ }^{2}$, mesmo comparando vários níveis $(0,0,15 ; 0,45 ; 0,75$; 1,$05 ; 1,35$ ppm), não observaram efeito em nenhuma idade. Resultados similares ao encontrado no presente estudo foram obtidos por Jianhua, Ohtsuka e Kunioki ${ }^{18}$ comparando 0,3 com 0,5 mg de Se por quilo de ração, onde o maior nível proporcionou o maior ganho de peso.

Apesar de não terem sido observadas diferenças estatísticas no consumo de ração, a conversão alimentar foi influenciada pela interação dos fatores fonte e nível de $\mathrm{Se}(\mathrm{p}=0,0198)$. Como resultado desta interação pode ser observado que ao nível de 0,45 $\mathrm{mg} / \mathrm{kg}$ não houve diferenças entre as fontes submetidas ao teste de média. Já ao nível de $0,15 \mathrm{mg} / \mathrm{kg}$ a fonte inorgânica acarretou melhor resultado que a orgânica, porém não diferindo da fonte In-org, sendo que esta última obteve valor intermediário não diferindo das demais. Este resultado discorda do encontrado por Moreira et al. ${ }^{2}$ e Dahlke et al. ${ }^{17}$ que não obtiveram interação entre fonte e nível. Edens ${ }^{7}$ 
Tabela 2 - Peso médio, ganho de peso médio (GP), ganho médio diário (GMD), consumo de ração e conversão alimentar (CA) aos 42 dias de idade

\begin{tabular}{|c|c|c|c|c|c|c|}
\hline \multicolumn{2}{|c|}{ Dietas experimentais } & \multicolumn{5}{|c|}{ Variáveis de Desempenho } \\
\hline Nível & Fonte & Peso & GP & GMD & Cons. & CA \\
\hline \multirow{3}{*}{0,15} & Inorgânico & 2884,23 & 2836,84 & 67,54 & 4867,54 & $1,72 \mathrm{~B}$ \\
\hline & Orgânico & 2824,60 & 2777,39 & 66,13 & 5032,98 & $1,81 \mathrm{~A}$ \\
\hline & In-org. & 2906,11 & 2859,24 & 68,08 & 5025,64 & $1,76 \mathrm{AB}$ \\
\hline \multirow{3}{*}{0,45} & Inorgânico & 2919,49 & 2872,35 & 68,39 & 4980,86 & $1,73 \mathrm{~A}$ \\
\hline & Orgânico & 2937,54 & 2889,77 & 68,80 & 4948,38 & $1,71 \mathrm{~A}$ \\
\hline & In-org. & 2936,61 & 2889,54 & 68,80 & 5057,22 & $1,75 \mathrm{~A}$ \\
\hline \multicolumn{7}{|c|}{ Efeitos Principais } \\
\hline 0,15 & & $2871,65 B$ & $2824,49 \mathrm{~B}$ & $67,25 B$ & 4975,39 & 1,76 \\
\hline \multirow[t]{4}{*}{0,45} & & $2931,21 \mathrm{~A}$ & $2883,89 \mathrm{~A}$ & $68,66 \mathrm{~A}$ & 4995,48 & 1,73 \\
\hline & Inorgânico & 2901,86 & 2854,59 & 67,97 & 4924,20 & 1,73 \\
\hline & Orgânico & 2921,36 & 2833,58 & 67,47 & 4990,68 & 1,76 \\
\hline & In-org. & 2881,07 & 2874,39 & 68,44 & 5041,43 & 1,75 \\
\hline Média & & 2901,43 & 2854,19 & 67,96 & 4985,44 & 1,75 \\
\hline $\mathrm{CV}(\%)$ & & 2,7 & 2,8 & 2,8 & 2,8 & 3,3 \\
\hline \multicolumn{7}{|c|}{ Probabilidade } \\
\hline & & 0,0225 & 0,0225 & 0,0225 & 0,6612 & 0,0828 \\
\hline & & 0,4243 & 0,4128 & 0,4128 & 0,1244 & 0,1810 \\
\hline & & 0,3259 & 0,3286 & 0,3286 & 0,2187 & 0,0198 \\
\hline
\end{tabular}

Médias seguidas de letras diferentes na mesma coluna diferem pelo teste de Tukey $(\mathrm{P}<0,05)$.

CV (\%): Coeficiente de variação.

também observou que a fonte orgânica de Se aumenta um pouco a conversão alimentar. Laganá et al. ${ }^{19}$ obtiveram melhor conversão alimentar quando utilizaram fonte orgânica, contrariando os resultados obtidos no presente estudo.

Aos 42 dias a conversão alimentar foi a única variável que apresentou interação entre fonte e nível de Se, sendo que as variáveis de peso somente apresentaram efeito do nível de inclusão do Se, mostrando que fonte e nível de Se são fatores que juntos podem exercer influência sobre o desempenho dos animais.

\section{Referências}

1.CHOCT, M.; NAYLOR, A. J.; REINKE, N. Selenium supplementation affects broiler growth performance, meat yield and feather coverage. British Poultry Science, v. 45, n. 5, p. 677-683, 2004.

2.MOREIRA, J.; SANTOS, C. D.; ABREU, C. M. P.; BERTECHINI, A. G.; OLIVEIRA, D. F.; CARDOSO, M. G. Efeito de fonte e níveis de selênio na atividade enzimática da glutationa peroxidase e no desempenho de frangos de corte. Ciência Agrotecnica, v. 25, n. 3, p. 664-666, 2001.

3.BERTECHINI, A. G.; FASSANI, E. J. Macro e microminerais na

\section{Conclusão}

Quanto ao desempenho ao final do ciclo de criação é possível concluir que o nível de inclusão de Se na dieta interfere no ganho de peso médio, ganho médio diário de peso e no peso médio. A interação entre fonte e nível de Se pode alterar uma das variáveis mais importantes da produção que é a conversão alimentar.

\section{Agradecimento}

À Fundação de Amparo à Pesquisa do Estado de São Paulo (FAPESP) pelo suporte financeiro (processo Fapesp 06/58491-6).

alimentação animal. In: SIMPÓSIO SOBRE INGREDIENTES NA ALIMENTAÇÃO ANIMAL, 1, 2001, Campinas. Anais... Campinas: Colégio Brasileiro de Nutrição Animal, 2001. p. 219-234.

4.RUTZ, F.; ANCIUTI, M. A.; RECH, J. L.; RECH, C. L. S.; ROSSI, P. Impacto da utilização de minerais orgânicos sobre o metabolismo e desempenho das aves. In: CONFERÊNCIA APINCO DE CIÊNCIA E TECNOLOGIA AVÍCOLAS, 2005 Santos. Anais... Campinas: FACTA, 2005. p. 257-268.

5.LEESON, S.; SUMMERS, J. D. Scott's nutrition of the chicken. 
Guelph: University Books, 2001. 591 p.

6. MAHAN, D. Organic selenium: using nature's model to redefine selenium suplementation for animals. In: ALLTECH'S ANNUAL SYMPOSIUM, 15., 1999, Nottingham, UK. Proceedings... Nottingham, UK: Nottingham University Press, 1999. p. 523-535.

7.EDENS, F. W. Organic selenium: from feather to muscle integrity to drip loss. In: LYONS, T. P.; JACQUES, K. A. Biotechnology in the Feed Industry. In: ANNUAL SYMPOSIUM, 12., 1996, Nottingham, UK. Proceedings... Nottingham, UK: Nottingham University Press, 1996. p. 165185.

8. ROCK, M. J.; KINCAID, R. L.; CARSTENS, G. E. Effect of prenatal source and level of dietary selenium on passive immunity and thermometabolism of newborn lambs. Small Ruminant Research, v. 40, n. 2, p. 129-138, 2001.

9.HEGAZY, S. M.; ADACHIT, Y. Compaison of the effects of dietatry selenium, zinc, and selenium and zinc suplementation on growth and immune response between chick groups that were inoculated with Salmonella and aflatoxim or Salmonella. Poultry Science, v. 79, n. 3, p. 331-335, 2000.

10.SURAI, P. F. Natural antioxidants in avian nutrition and reproduction. Nottingham: Nottingham University Press, 2002. 790 p.

11.MAHMOUDM, K. Z; EDENS, F. W. Influence of organic selenium on hsp70 response of heat-stressed and enteropathogenic Escherichia coli-challenged broiler chickens (Gallus gallus). Comparative Biochemistry and Physiology, v. 141, n. 1, p. $69-75,2005$.

12.SCHRAUZER, G. N. Selenomethionine: a review of its nutritional significance, metabolism and toxicity. Journal of
Nutrition, v. 130, n. 7, p. 1653-1656, 2000.

13.BRITISH NUTRITION FOUNDATION. Selenium and health. London: British Nutrition Foundation, 2001. 48 p.

14.PAN, C.; HUANG, K.; ZHAO, Y.; QIN, S.; CHEN, F.; HU, Q. Effect of selenium source and level in hen's diet on tissue selenium deposition and egg selenium concentrations. Journal of Agricultural and Food Chemistry, v. 55, n. 3, p. 1027-1032, 2007.

15.YOON, I.; WERNER, T. M.; BUTLER, J. M. Effect of source and concentration of selenium on growth performance and selenium retention in broiler chickens. Poultry Science, v. 86, n. 4, p. 727-730, 2007

16.SWAIN, B. K.; JOHRI, T. S.; MAJUMDAR, S. Effect of supplementation of vitamin E, selenium and their different combinations on the performance and immune response of broilers. British Poultry Science, v. 41, n. 3, p. 287-292, 2000 .

17.DAHLKE, F.; GONZALES, E.; FURLAN, R. L.; GADELHA, A. C.; MAIORKA, A.; ALMEIDA, J. G. Avaliação de diferentes fontes e níveis de selênio para frangos de corte em diferentes temperaturas. Archives of Veterinary Science, v. 10, n. 1, p. 21-26, 2005.

18.JIANHUA, H.; OHTSUKA, A.; KUNIOKI, H. Selenium influences growth via thyroid hormones status in broilers chickens. British Journal of Nutrition, v. 84, n. 5, p. 727-732, 2000.

19.LAGANÁ, C.; RIBEIRO, A. M. L.; KESSLER, A. M.; KRATZ, L. R.; PINHEIRO, C. C. Effect of the supplementation of vitamins and organic minerals on the performance of broilers under heat stress. Brazilian Journal of Poultry Science, v. 9, n. 1, p. 39-43, 2007. 